\title{
La implementación de indicadores de lo intangible para catalogar el paisaje percibido. El caso del Observatorio del Paisaje de Cataluña'
}

\author{
Joan Nogué2, Jordi de San Eugenio ${ }^{3}$ y Pere Sala ${ }^{4}$
}

\begin{abstract}
RESUMEN
La definición de paisaje impulsada por el Convenio Europeo del Paisaje (2000), enfatiza los valores simbólicos que la ciudadanía atribuye al paisaje en función de cómo éste es percibido. En este sentido, el gobierno de Catalunya impulsó la Ley de Protección, Gestión y Ordenación del Paisaje (2005), que contemplaba, entre otras acciones, la evaluación transversal (física y simbólica) de los paisajes de Catalunya mediante el establecimiento de los denominados como 'Catálogos de Paisaje'. Por todo lo expuesto, este artículo tiene por objetivo el análisis, a nivel teórico-aplicado y mediante la metodología del estudio de caso, de los indicadores de paisaje implementados por el Observatorio del Paisaje de Cataluña, haciendo especial hincapié en el despliegue de indicadores intangibles, a razón de que esta iniciativa supone, per se, una apuesta inédita en Europa en el establecimiento de una catalogación de los valores inmateriales de un paisaje por parte de un ente público.
\end{abstract}

Palabras clave: indicadores de paisaje, paisaje intangible, Observatorio del Paisaje de Cataluña, catálogos de paisaje.

\begin{abstract}
The definition of landscape promoted by the European Landscape Convention (2000), emphasizes the symbolic values that citizens attribute to the landscape in terms of how it is perceived. In that sense, the Catalan government promoted the Landscape Protection, Management and Planning Act (2005), which, among other actions, included the physical and symbolic evaluation of landscapes in Catalonia through establishment of the so-called 'Landscape Catalogues'. According to this, the aim of this article is to analyse, at a theoretical and applied level and through the study case methodology, the landscape indicators implemented by the Landscape Observatory of Catalonia, with special emphasis on the development of intangible indicators, since this initiative represents an unprecedented project in Europe with regard to a public body establishing a catalogue of intangible values for a given landscape.
\end{abstract}

Keywords: landscape indicators, intangible landscape, Landscape Observatory of Catalonia, landscape catalogues.

Artículo recibido el 17 de abril de 2018, aceptado el 29 de agosto de 2018 y corregido el 3 de octubre de 2018 Departmento de Geografia, Universitat de Girona (España). E-mail: joan.nogue@udg.edu

Departamento de Comunicaciones, Universitad de Vic-Universitad Central de Cataluña (España). E-mail: jordi.saneugenio@uvic.cat Observatorio del Paisaje de Cataluña (España).E-mail: pere.sala@catpaisatge.net 
El establecimiento de indicadores resulta fundamental en la concreción de una métrica del paisaje que permita, de forma paralela, identificar sus valores tangibles e intangibles, a la vez que dotarse de argumentos que permitan mejorar las actuales políticas de gestión del paisaje (Sala, 2009; Zaragozí et al., 2012). Aunque la noción de paisaje es relativamente reciente, parece claro que el término paisaje abraza conceptos que vinculan a un determinado espacio natural con la observación y posterior interpretación humana que le resulta inherente e irrenunciable. El paisaje deviene, en esta tesitura, un fiel reflejo de la interacción constante que se produce entre el medio natural y la acción humana (Nadal, 2009; Nogué y San Eugenio, 2011).

En los últimos años el concepto de paisaje está recibiendo aportaciones destacadísimas provenientes de América Latina, desde todos los ámbitos disciplinarios. No podemos detallar todas estas aportaciones porque nos alargaríamos en exceso, pero sí vamos a poner algunos ejemplos que muestran esta vitalidad extraordinaria. Desde la ecología del paisaje, por ejemplo, destacaríamos las contribuciones de los congresos organizados regularmente por parte de la Asociación Argentina de Ecología de Paisajes, como el recientemente celebrado en 2017 en la Universidad Nacional de Santiago del Estero (Lorenz et al., 2017). Estos congresos vienen celebrándose periódicamente desde el año 2005, cuando se celebró el primero en Buenos aires.

Desde el ámbito de la planificación del territorio las aportaciones son también muy relevantes e innovadoras. Desde México los geógrafos Martín M. Checa-Artasu y Pere Sunyer Martín acaban de publicar una interesante contribución sobre paisaje y ordenamiento territorial (Checa-Artasu y Sunyer Martín, 2018). No podemos pasar por alto en esta cuestión la aparición en el año 2012 de la Iniciativa Latinoamericana del Paisaje (LALI, por las iniciales de su nombre en inglés), impulsada desde Colombia sobre todo por parte de arquitectos y paisajistas. La LALI propone convenir políticas binacionales y multinacionales en materia de conservación, protección, gestión y recuperación de unidades de paisaje ubicadas en zonas transfronterizas, planteándose también, de caras al futuro, una Convención Internacional del Paisaje. Otro de los documentos de relevancia en el debate latinoamericano actual sobre la intervención en el paisaje lo constituye la Carta Iberoamericana del Paisaje Cultural (CIPC), cuyas recomendaciones cristalizaron en un encuentro en Cartagena de Indias en noviembre de 2012. Este documento persigue la unificación de criterios para la identificación, reconocimiento, protección y formulación de metodologías de actuación sobre el patrimonio paisajístico. Asimismo, considera la dimensión histórica y los rasgos distintivos como fundamento de su salvaguarda y fortalecimiento, como recuerdan Pastor et al. (2016). La cuestión del paisaje como patrimonio o, si se quiere, el tema de la patrimonialización del paisaje está recibiendo también interesantes aportaciones, en especial desde Chile (Valdés, 2012; Valencia, 2017; Booth y Valdés, 2016).

El paisaje como construcción social es uno de los conceptos y líneas de trabajo que, a nuestro entender, más frutos está dando en América Latina en general. Son constantes las aportaciones en este sentido (Penhos, 2016; Silvestri, 2011), incorporando algunas de ellas una dimensión geopolítica y cultural vinculada a la construcción de imaginarios geográficos de una extraordinaria relevancia (Zusman, 2000; Núñez et al., 2013; Núñez et al., 2017). Por su parte, y a partir de una mirada que pone el énfasis en las relaciones sociales como agente territorializador, Pastor et al. (2016) describen las nociones de paisaje subyacentes, así como las perspectivas teóricas que sostienen algunas de las instrumentaciones para aprehender y reconocer qué paisajes hemos producido y estamos produciendo socialmente. 
Así pues, muchas y diversas están siendo las aproximaciones al concepto de paisaje en todo el mundo, pero, grosso modo, podemos agruparlas en dos grandes bloques. Por un lado, las provenientes del ámbito de los estudios humanísticos, culturales y semióticos, que tienden a concebir el paisaje exclusivamente como una construcción social y cultural. Por otro lado, encontramos las aproximaciones propias de algunas metodologías desplegadas en el ámbito de las ciencias naturales, de base mucho más emprírica y materialista. Los autores del presente artículo pensamos que hay que huir de esta polarización y apostar por una concepción del paisaje integral que, por un lado, resalte su singularidad geohistórica -todo paisaje pertenece a un espacio y a un tiempo concretos en el sentido más materialista de ambos términos- $y$, por otro lado, nunca olvide que este hecho geohistórico puede ser objeto -será siempre objeto- de diferentes miradas, percepciones, interpretaciones y lecturas. Son encomiables las aproximaciones al paisaje que parten de un análisis fisionómico y morfológico para llegar a una descripción-caracterización de sus elementos, en una primera fase y, aunque no siempre, a la comprensión sistémica de sus elementos, en una segunda fase. Igualmente, notables son las aproximaciones que se centran en entender el paisaje como una construcción social y cultural. Ahora bien, ambas aproximaciones, por separado, quedan cojas, porque, en efecto, el paisaje es una fuente de información de carácter material y tangible extraordinaria, pero es también un universo de sensaciones intangibles, no siempre visibles y a menudo efímeras. Así pues, el paisaje es, a la vez, una realidad física y la representación que culturalmente nos hacemos de ella; la fisonomía externa y visible de una determinada porción de la superficie terrestre y la percepción individual y social que genera; un tangible geográfico y su interpretación intangible. Es, también, el significado y el significante, el continente y el contenido, la realidad y la ficción, el original y la copia (Nogué, 2009). Por su parte, y en una línea muy similar, la Convención Europea del Paisaje (CEP) define el paisaje como "un área, tal y como la percibe la población, el carácter de la cual es el resultado de la acción y la interacción de factores naturales y/o humanos" (Consejo de Europa, 2000). Es precisamente esta dualidad, es decir el reconocimiento de que el paisaje puede generar, simultáneamente, emociones individuales y ser depositario de valores científicamente reconocidos y socialmente consensuados, lo que enriquece la temática del paisaje y nos lleva un poquito más allá para afirmar que la aceptación de esta dimensión más individual y subjetiva del paisaje no es incompatible con la gestión colectiva -es decir, pública- de sus valores, una vez identificados, caracterizados y consensuados democráticamente.

Y aquí es precisamente cuando entran con fuerza y gran legitimación los indicadores de paisaje. Como indica Priore (2009), hoy resulta imprescindible avanzar hacia aspectos relacionados con la evaluación de la percepción que la población tiene de sus paisajes mediante el previo establecimiento de indicadores. En este sentido, la relación entre indicadores y paisaje puede establecerse en base a la creciente necesidad de seguir periódicamente la evolución y estado de los paisajes, de medir la satisfacción de la población con su entorno o bien para valorar la efectividad de las iniciativas públicas y privadas en la mejora continua y global del paisaje.

Por tanto, lo que en este trabajo se pretende analizar tiene como punto de partida la definición de paisaje ofrecida por el Convenio Europeo del Paisaje, ya que presupone el diseño de nuevas herramientas, concretadas en indicadores, que permitan ir más allá de la evaluación cuantitativa o cualitativa de elementos paisajísticos materiales, para pasar a abordar, con plena responsabilidad, las legítimas aspiraciones de la ciudadanía en lo referido a las características percibidas de sus entornos vitales. Ello obliga a cambiar inercias históricas vinculadas a la gestión pública del 
paisaje, y más concretamente, en lo que atañe a la definición de nuevos marcos de trabajo que permitan una nueva adaptación a la diversidad de percepciones y apreciaciones que sobre el paisaje nos ofrece la población.

Sin embargo, el debate sobre la naturaleza de los indicadores de paisaje subyace en Europa, especialmente por la complejidad inherente al establecimiento de métricas relacionadas con la transversalidad del propio concepto, que engloba dimensiones naturales y culturales, así como una variable perceptiva individual y social (Lang et al., 2008). Además, el paisaje es relativamente nuevo en su consideración de elemento de ordenación del territorio y del planeamiento urbanístico. En suma, se constata la necesidad de disponer de una lista de indicadores rigurosos que sean aplicables y comprensibles para la sociedad en su conjunto (Nogué, 2009). Por todo ello, este trabajo va a analizar los indicadores de paisaje implementados por el Observatorio del Paisaje de Cataluña, haciendo énfasis en el indicador en sí, con un acento especial en la voluntad de este ente por establecer indicadores que sean capaces de catalogar aspectos perceptivos/subjetivos del paisaje.

\section{Los indicadores y su transposición al paisaje}

Gallopin (2006: 12) define al indicador como "un parámetro, una mesura, un valor, una fracción, una fracción comparando una cantidad (numerador) con una mesura científica o arbitraria escogida (denominador), un índice, un componente de un índice, algo, una porción de información, una cantidad simple que deriva de una variable y se utiliza para reflejar un atributo, un modelo empírico de la realidad, un signo". En cualquier caso, este mismo autor señala la existencia de dos tipologías básicas de indicadores. Por un lado, los indicadores que son reflejo de actuaciones pasadas, ya que ofrecen información sobre lo que ya ha pasado, sin que haya posibilidad de cambiar el resultado. Por el otro, los indicadores que describen lo que se hace (ejecución), los cuales son generalmente de corta duración y se conocen como inductores. Este tipo de indicadores detectan lo que está pasando y emprenden acciones apropiadas para mejorar el resultado.

En cualquier caso, se puede convenir que cualquier indicador debe reflejar con claridad qué se debe medir, como hacerlo, cuanto medir, la fuente de medición y el responsable de la medición. Por tanto, los indicadores deben ser variables críticas definidas en relación a un objetivo, se pueden referir a acciones (pasadas o futuras), no a situaciones indeterminadas y que comporten mesura (Bru, 2009).

Aun así, existe un elemento añadido de complejidad que da sentido al estudio de caso que aquí se quiere plantear. Tomando prestado el criterio de Folch (1999), se trata, en definitiva, de compatibilizar lo que per se, representa un indicador, esto es, un parámetro más o menos vectorializado, o, en cualquier caso, una correlación entre dos parámetros tomados de tal forma que doten de significado a una información cuantitativa, capaz de tener sentido cualitativo. El difícil equilibrio que se desprende de una relación históricamente compleja entre los elementos cuantitativos y cualitativos que posee cualquier indicador, sitúan a éstos en una disyuntiva simbólica que implica una relación compleja, quizá borrosa (fuzzy) con la antinomia entre objetividad y subjetividad. Ello no implica, en ningún caso, que los indicadores no puedan ser, o bien no deban ser objetivos (Bru, 2009).

Lo que sí debe tenerse en cuenta para afrontar el estudio de caso que se planteará a lo largo de este artículo, es que los indicadores disponen de una sobrecarga simbólica poco precisa, 
que, si bien nos posibilitan el descubrimiento de determinados aspectos de la realidad, ésta no puede ser leída en todo su alcance por el hecho de estar regida por una determinada mirada irremediablemente subjetiva, esto es, lejos de la falsa ilusión de la 'pura objetividad' asociada habitualmente a los índices. En este sentido, debemos situarnos cerca de la tesis de Cobb y Rixford (1998) quienes señalan que los indicadores se han desempeñado siempre a modo de intento de cambiar algún resultado de la realidad, y más aún, como una materialización de la vieja aspiración de dirigir la sociedad sobre bases racionales.

En lo referido a la vinculación entre indicadores y paisaje, el diccionario de Medio Ambiente y Conservación (Park \& Allaby, 2017) editado por Oxford University Press define el indicador de paisaje como "un índice o medida de algún aspecto del paisaje de un lugar particular (como la extensión de una cubierta forestal), basado en datos cartografiados o teledetectados, que puede utilizarse para describir el uso de la tierra y monitorear el cambio ambiental". Asimismo, Dale \& Kline (2013: 91) entienden que los indicadores de paisaje "son medidas del tamaño, forma y yuxtaposición de determinados tipos de terrenos, así como la configuración de todos los tipos de tierra dentro de un área".

En este sentido, y durante muchos años, ha sido la ecología del paisaje ${ }^{5}$ quién se ha ocupado de establecer una métrica del paisaje ${ }^{6}$ a partir de una previa definición de indicadores, con el fin de describir, sistemáticamente, sus patrones de comportamiento, además de predecir los efectos resultado de los cambios de patrones en diferentes especies (Riitters et al., 1995; Burel \& Baudry, 2003; Farina, 2006; Llausàs y Nogué, 2012). De este modo, se han desarrollado métricas de paisaje para cuantificar la composición y configuración de los elementos del paisaje (O'Neill et al., 1999; Turner et al., 2001) y para describir los cambios en el paisaje (como la fragmentación de los bosques) y su funcionalidad (Dale \& Kline, 2013). Esta métrica del paisaje ha fundamentado su definición de indicadores a partir de variables esencialmente cuantitativas, requisito previo e indispensable para el establecimiento de patrones de paisaje a partir de los cuales vincular decisiones de gestión (Turner et al., 2001; Uuemaa et al., 2013).

Sin embargo, la definición de paisaje impulsada por el Consejo de Europa, y más concretamente, por el Convenio Europeo del Paisaje ${ }^{7}$ (2000), destaca la importancia de los elementos perceptivos, esto es, la necesidad de establecer elementos de catalogación del paisaje como realidad percibida. Esta perspectiva integradora y transversal del paisaje tiene en cuenta, además de los tradicionales aspectos ecológicos del paisaje vinculados a su gestión y planificación, aspectos históricos y características sociales del territorio, lo que hace deseable, por no decir necesaria, la incorporación de aspectos vinculados a la percepción y posterior valorización del paisaje por parte de la ciudadanía a cualquier evaluación, monitorización o bien en escenarios de toma de decisiones que impliquen cambios en el paisaje (Llausàs y Nogué, 2012).

\footnotetext{
Podemos definir la landscape ecology como una visión holística de la realidad que intenta integrar al máximo su extremada y dinámica complejidad. Una visión de síntesis fundamentada especialmente en la incorporación, por una parte, de la interpretación de la heterogeneidad horizontal, un enfoque propio de la geografía que centra su atención en la distribución de los paisajes a lo largo del territorio. Y, por otra parte, el análisis de la heterogeneidad vertical, una perspectiva propia de la ecología, que hace hincapié en la interrelación entre los distintos elementos bióticos y abióticos en una porción determinada de paisaje (Vila et al., 2006: 154-155).

6 A partir de técnicas como el paisaje-mosaico de la cubierta vegetal, el establecimiento de gradientes para la copa de los árboles o bien métricas de superficie de degradado del paisaje, entre otras (Frazier \& Kedron, 2017).

Cualquier parte del territorio tal como la percibe la población, cuyo carácter sea el resultado de la acción y la interacción de factores naturales y/o humanos. Disponible en Internet: http://ipce.mcu.es/pdfs/convencion-florencia.pdf
} 
He aquí uno de los retos mayúsculos que la evaluación holística del paisaje ha planteado en los últimos tiempos: la combinación de indicadores cuantitativos (vinculados a aspectos ecológicos) e indicadores cualitativos (vinculados a la percepción humana) que permitan definir las características materiales e inmateriales de un determinado paisaje en toda su extensión. En la misma dirección, muchos autores han reconocido la necesidad de integración de las dimensiones ecológicas y perceptivas en un solo indicador, o bien en una combinación de indicadores (Farina, 2006).

Los aspectos vinculados al paisaje subjetivo, esto es, el paisaje vivido por la ciudadanía, se han asignado a un ámbito de conocimiento denominado 'estética del paisaje', ocupada en estudiar el paisaje cultural y la 'apreciación estética/simbólica' del paisaje vivido y/o percibido (Nogué, 2009). La estética del paisaje, en oposición a la ecología del paisaje, representa, entonces, un intento de transformación hacia lo objetivo de lo sumamente subjetivo (percepción del paisaje). Lo que sí parece claro es que las actuales métricas de paisaje no permiten medir todos los aspectos visuales del paisaje, lo que hace indispensable la monitorización de los valores estéticos del paisaje. Existe, en esta línea, un profundo vacío de estudios con objetivos orientados hacia una métrica que incorpore la evaluación de diferentes aspectos del carácter visual del paisaje tales como la naturalidad o la diversidad (Uuemaa et al., 2013). Es por todo ello que nos parece especialmente relevante el estudio de caso del Observatorio del Paisaje de Cataluña que se va a abordar a continuación. Por tanto, y en términos metodológicos, esta investigación ha optado por el estudio de caso, que se concentra, según Yacuzzi (2005), en el método como forma de generar teorías sobre fenómenos sociales y organizacionales de causalidad compleja.

\section{El caso del Observatorio del Paisaje de Cataluña}

Con el objetivo de ofrecer una salida efectiva a los nuevos retos planteados en la evaluación integral del paisaje, especialmente en lo referido al análisis y posterior catalogación de sus valores y/o atributos intangibles, el Observatorio del Paisaje de Cataluña promueve, desde 2006, los Catálogos del Paisaje ${ }^{8}$, que son resultado de la Ley 8/2005, de 8 de junio, de Protección, Gestión y Ordenación del Paisaje de Cataluña ${ }^{9}$. En esta tesitura, los catálogos devienen nuevos instrumentos para la introducción de objetivos paisajísticos en la planificación territorial de Cataluña, así como en las políticas sectoriales, adoptando, de este modo, los principios y estrategias de acción que establece el Convenio Europeo del Paisaje. Asimismo, esta catalogación de los paisajes de Cataluña, se produce en base a una previa definición de indicadores, concebidos como instrumentos al servicio de las políticas de paisaje (Sala, 2009).

El estudio de caso que se representa a continuación, tiene, a nuestro entender, especial relevancia a tenor de una disposición inédita y pionera de indicadores de paisaje que se conciben con la voluntad inicial de inventariar los paisajes de Cataluña a nivel material e inmaterial. Con tal finalidad, se habilitan instrumentos de medición de sus características físicas ya usados tradicionalmente para estos menesteres, aunque también se consideran las representaciones de paisaje provenientes de la población, basadas en una gran variedad de características físicas y materiales, pero también de vínculos culturales, emocionales y espirituales (Sala, 2009).

\footnotetext{
Son las herramientas que permiten conocer cómo es el paisaje de Cataluña y qué valores tiene, qué factores explican un determinado tipo de paisaje y no otro, cómo evoluciona el paisaje en función de las actuales dinámicas económicas, sociales y ambientales y, finalmente, definen qué tipo de paisaje se quiere y cómo conseguirlo. Fuente: Observatorio del Paisaje de Cataluña. Disponible en Internet: http://www.catpaisatge.net/esp/catalegs_documentacio.php

Disponible en Internet: https://www.boe.es/buscar/act.php?id=BOE-A-2005-11753
} 


\section{Funciones de los indicadores del paisaje de Cataluña}

Siguiendo a Sala (2009: 115), los indicadores del paisaje de Cataluña deben orientarse hacia tres ámbitos de acción fundamentales:

a) Descripción: los indicadores deben describir, de forma simplificada pero rigurosa, la realidad paisajística de Cataluña, contribuyendo a identificar los problemas, facilitar el conocimiento de los retos existentes en materia de conservación, gestión y ordenación del paisaje, y permitir la búsqueda y obtención de soluciones adecuadas y flexibles.

b) Evaluación: se trata de evaluar la efectividad de la actuación de los diversos niveles de Administración en el ámbito del paisaje o proporcionar señales de aviso sobre el éxito o el fracaso de las políticas adoptadas, anticipando futuras condiciones y tendencias.

c) Comunicación: los indicadores de paisaje deben comunicar a los ciudadanos sobre los aspectos paisajísticos de una manera comprensible y precisa, facilitando y mejorando su comprensión. En este punto vale la pena destacar que los indicadores de paisaje deben contribuir, además, a concienciar y educar a la ciudadanía. Esta legibilidad favorecerá la participación.

Figura $\mathrm{N}^{\circ} 1$

Funciones de los indicadores de paisaje de Cataluña

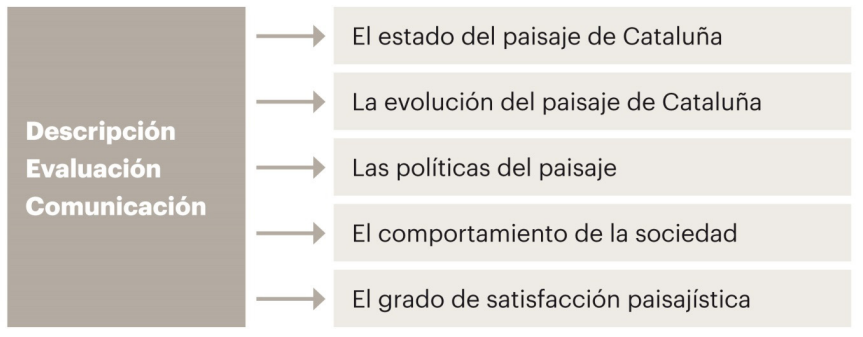

Fuente: Elaboración propia a partir de Sala (2009).

\section{Los indicadores de paisaje de Cataluña}

A continuación, se van a exponer, sintéticamente, los diez indicadores de paisaje impulsados por el Observatorio del Paisaje de Cataluña.

La revisión profunda de la literatura existente referida a indicadores de paisaje ha permitido constatar que no existe un consenso generalizado en torno a una lista de indicadores común y universalmente homologada. Según Sala (2009), ello se explica porque el paisaje, y más concretamente, su protección, gestión y ordenación, representa un ámbito en plena fase de desarrollo normativo y técnico. Sin embargo, existen otros tipos de indicadores, como por ejemplo los relacionados con la evaluación de la biodiversidad, los ambientales o los vinculados al desarrollo sostenible, que cuentan con un bagaje mucho más amplio. 
Cuadro $\mathrm{N}^{\circ} 1$

Los indicadores de paisaje de Cataluña

\begin{tabular}{|c|c|c|c|}
\hline INDICADOR & DESCRIPCIÓN & NATURALEZA & $\begin{array}{l}\text { ÁMBITO DE } \\
\text { CONOCIMIENTO }\end{array}$ \\
\hline $\begin{array}{l}\text { Transformación } \\
\text { del paisaje }\end{array}$ & $\begin{array}{l}\text { Análisis del cambio en las } \\
\text { características naturales } \\
\text { o culturales del paisaje } \\
\text { tendiendo a la modificación } \\
\text { de sus valores o su } \\
\text { apariencia }\end{array}$ & Paisaje físico/tangible & Ecología del paisaje \\
\hline Diversidad paisajística & $\begin{array}{l}\text { Evolución de la riqueza en } \\
\text { configuraciones y caracteres } \\
\text { paisajísticos, ya sea a través } \\
\text { de elementos como de } \\
\text { paisajes en su conjunto }\end{array}$ & Paisaje físico/tangible & Ecología del paisaje \\
\hline $\begin{array}{l}\text { Fragmentación } \\
\text { paisajística }\end{array}$ & $\begin{array}{l}\text { Resultado de un proceso de } \\
\text { ruptura de la continuidad de } \\
\text { un paisaje y su coherencia. } \\
\text { Puede ser de tipo territorial, } \\
\text { ecológica, social y visual. }\end{array}$ & Paisaje físico/tangible & Ecología del paisaje \\
\hline $\begin{array}{l}\text { Valor económico } \\
\text { del paisaje }\end{array}$ & $\begin{array}{l}\text { Capacidad de un paisaje } \\
\text { para convertir sus elementos } \\
\text { constituyentes en recursos } \\
\text { productivos de diferente } \\
\text { valía económica }\end{array}$ & Paisaje físico/tangible & $\begin{array}{l}\text { Ecología del paisaje/ } \\
\text { Economía territorial }\end{array}$ \\
\hline $\begin{array}{l}\text { Conocimiento } \\
\text { del paisaje }\end{array}$ & $\begin{array}{l}\text { Grado de formación y } \\
\text { reconocimiento del paisaje } \\
\text { que tiene y experimenta una } \\
\text { población determinada }\end{array}$ & Paisaje simbólico/intangible & $\begin{array}{l}\text { Geografía de la Percepción } \\
\text { y del Comportamiento } \\
\text { Ambiental/Geografía } \\
\text { Humanística/Geografía } \\
\text { Emocional }\end{array}$ \\
\hline Satisfacción paisajística & $\begin{array}{l}\text { Expresión del grado } \\
\text { de contentamiento o } \\
\text { descontentamiento de } \\
\text { la población que vive en } \\
\text { un determinado ámbito } \\
\text { territorial con su paisaje }\end{array}$ & Paisaje simbólico/intangible & $\begin{array}{l}\text { Ecología del paisaje/ } \\
\text { Geografía de la Percepción } \\
\text { y del Comportamiento } \\
\text { Ambiental/Geografía } \\
\text { Humanística/Geografía } \\
\text { Emocional }\end{array}$ \\
\hline Sociabilidad paisajística & $\begin{array}{l}\text { Permite calibrar las } \\
\text { relaciones sociales en su } \\
\text { sentido amplio vinculadas al } \\
\text { paisaje y generadas por el } \\
\text { paisaje }\end{array}$ & Paisaje simbólico/intangible & $\begin{array}{l}\text { Geografía de la Percepción } \\
\text { y del Comportamiento } \\
\text { Ambiental/Geografía } \\
\text { Humanística/Geografía } \\
\text { Emocional }\end{array}$ \\
\hline $\begin{array}{l}\text { Comunicación } \\
\text { en el paisaje }\end{array}$ & $\begin{array}{l}\text { Transmisión de significados } \\
\text { culturales en relación a uno o } \\
\text { más paisajes a través de los } \\
\text { medios y las tecnologías de } \\
\text { la información }\end{array}$ & Paisaje simbólico/intangible & $\begin{array}{l}\text { Ecología del paisaje/ } \\
\text { Geografía de la Percepción } \\
\text { y del Comportamiento } \\
\text { Ambiental/Geografía } \\
\text { Humanística/Geografía } \\
\text { Emocional }\end{array}$ \\
\hline $\begin{array}{l}\text { Actuación pública } \\
\text { y privada en la } \\
\text { conservación, gestión y } \\
\text { ordenación del paisaje }\end{array}$ & $\begin{array}{l}\text { Monitorización de las } \\
\text { políticas públicas y de las } \\
\text { actuaciones privadas en el } \\
\text { ámbito de la conservación, } \\
\text { gestión y ordenación del } \\
\text { paisaje }\end{array}$ & Paisaje físico/tangible & Ecología del paisaje \\
\hline $\begin{array}{l}\text { Aplicación de los } \\
\text { instrumentos de la Ley } \\
\text { de Paisaje } 8 / 2005 \text { de } 8 \\
\text { de junio }\end{array}$ & $\begin{array}{l}\text { Evaluación de la aplicación } \\
\text { de los instrumentos de la Ley } \\
\text { de Paisaje }\end{array}$ & Paisaje físico/tangible & Ecología del paisaje \\
\hline
\end{tabular}

Fuente: Elaboración propia a partir de Sala (2009). 
Aun así, parece claro que, actualmente, una pretendida evaluación transversal del paisaje requiere del impulso de indicadores que sitúen su foco de interés en el análisis de su dimensión inmaterial, asumiendo, con normalidad, elevados índices de complejidad e inexactitud.

Como se ha podido observar, la relación de indicadores (10) impulsada por el Observatorio de Cataluña contempla la evaluación tanto del paisaje físico como del paisaje simbólico. En este sentido, hasta 4 indicadores fijan su interés en la evaluación del paisaje percibido (conocimiento del paisaje, satisfacción paisajística, sociabilidad paisajística y comunicación en el paisaje). De igual forma, 6 de los indicadores impulsados por el Observatorio del Paisaje de Cataluña se ocupan de evaluar el paisaje material (transformación del paisaje, diversidad paisajística, fragmentación paisajística, valor económico del paisaje, actuación pública y privada en la conservación, gestión y ordenación del paisaje y, finalmente, aplicación de los instrumentos de la Ley de Paisaje 8/2005 de 8 de junio).

Metodológicamente, el Observatorio del Paisaje ha impulsado la participación de la población en la delimitación de los valores intangibles del paisaje mediante el despliegue de una consulta pública a tres niveles (Nogué y Sala, 2006, p. 28) ${ }^{10}$ :

a) Entrevista directa a agentes del paisaje, como instituciones, expertos, empresarios, sindicatos o asociaciones culturales y ecologistas.

b) Jornadas o sesiones de trabajo integradas en el proceso de consulta de los Planes Territoriales Parciales de la Generalitat de Cataluña.

c) Consulta a través del web del Observatorio a través de encuestas dirigidas a toda la sociedad.

En cuanto a los ámbitos de conocimiento vinculados a los indicadores, mientras que los aspectos más 'terrenales' del paisaje se vinculan a la ecología del paisaje, disciplina que, históricamente, y como ya se ha comentado, se ocupa de establecer algo cercano a una métrica cuantitativa de paisaje, los indicadores más próximos a lo 'espiritual', esto es, a lo perceptivo, intangible y/o simbólico, se ubican en el ámbito de conocimiento de la geografía de la percepción y del comportamiento ambiental, la geografía humanística y la geografía emocional, respectivamente.

Los estudios de percepción y comportamiento humanos en geografía pretenden aportar un valor añadido de explicación y predicción de los fenómenos de localización espacial de la ciudadanía. Esencialmente, propone la incorporación de la variable cognitiva o de percepción y vivencia geográfica del individuo, enfatizando, de este modo, la realidad subjetiva y perceptiva de la población (Tuan, 1976; Estébanez, 1989). A su turno, la geografía humanística adopta una entidad ideográfica o de investigación de valores (idios) en detrimento de la visión nomotética (leyes) propia de la geografía cuantitativa. En este contexto, la descripción, la explicación y la interpretación de la vivencia individual y colectiva del espacio geográfico representan su objeto de estudio principal (Tuan, 1976; Estébanez, 1989). Por último, la geografía emocional representa la búsqueda de los vínculos afectivos de las sociedades con sus espacios o bien el estudio de la emoción que proporciona la interacción humana con los lugares (Davidson et al., 2005).

Más información sobre la consulta pública desplegada por el Observatorio del Paisaje de Cataluña. Disponible en Internet: http://www.catpaisatge.net/esp/catalegs_participacio.php 


\section{La necesidad de catalogar el intangible paisajístico a través de indicadores y su efectiva implementación}

El paisaje se concibe como una realidad física y, al mismo tiempo, como la representación que de él se hace la población. Por tanto, un planteamiento de indicadores dirigidos a evaluar, únicamente, el paisaje físico, tangible o visible, por decirlo de algún modo, adolecería de un aspecto fundamental para evaluar sus características en toda su extensión: su dimensión subjetiva. El Observatorio del Paisaje de Cataluña, en la disposición de su genuina relación de indicadores de paisaje, tiene muy en cuenta la dimensión perceptiva del paisaje, entendiendo su complejidad constitutiva, por sus connotaciones de producto social, de proyección cultural de una sociedad en un espacio determinado desde una dimensión material, espiritual y simbólica. Ello, conlleva, sin duda alguna, dificultades metodológicas para definir un método cuantitativo y cualitativo de valoración de la calidad de un paisaje que sea válido y aceptado por la ciudadanía (Sala, 2009).

La efectiva implementación de los diez indicadores de paisaje (tangibles e intangibles) anteriormente enumerados, se produce en cuatro ámbitos de trabajo fundamentales (Sala 2009: 113-114):

a) Los indicadores están al servicio de las políticas de paisaje del Gobierno de Cataluña. Sirven para evaluar la eficacia de sus objetivos e iniciativas.

b) Resultan elementos clave en la confección de los siete Catálogos de Paisaje que emanan de la Ley de Paisaje 8/2005 de Cataluña. Se trata de documentos de carácter descriptivo y prospectivo para determinar las diferentes tipologías de paisaje de Cataluña, para identificar sus valores y el estado de conservación, además de proponer los objetivos de calidad paisajística que deben cumplir.

c) La redacción del informe sobre el estado del paisaje en Cataluña, que elabora el Observatorio del Paisaje cada cuatro años, se nutre, fundamentalmente, de una batería básica de los indicadores de paisaje anteriormente descritos.

d) Los objetivos de calidad paisajística para Cataluña que definió el Observatorio del Paisaje en 2007 están estrechamente vinculados a los indicadores del paisaje.

En cualquier caso, los indicadores para evaluar los aspectos intangibles del paisaje impulsados por el Observatorio del Paisaje de Cataluña han resultado clave para redactar los Catálogos del Paisaje, que, como se ha podido ver a lo largo del presente trabajo, representan un esfuerzo por inventariar, a la vez que evaluar, los paisajes existentes en Cataluña. Asimismo, el ejemplo más claro de implementación de los indicadores intangibles del paisaje se produce, a nuestro entender, en base a la definición de valores de paisaje ${ }^{11}$, esto es, resultado de la toma en consideración de los atributos patentes (físicos) y latentes (culturales, simbólicos y espirituales, entre otros), existentes en los paisajes catalanes, que se concretan y describen, de forma minuciosa, en los siete Catálogos del Paisaje elaborados por el Observatorio del Paisaje de Cataluña.

En este sentido, el Observatorio del Paisaje define ocho valores de paisaje a integrar en los Catálogos del Paisaje, la mayor parte de los cuales tienen por objetivo la determinación de sus atributos inmateriales (Nogué y Sala, 2006: 22-24):

La definición de valores inmateriales del paisaje pretende ofrecer una salida fiable a preguntas del tipo: ¿Cuánto vale una puesta de sol? 
a) Estético: capacidad que tiene un paisaje para transmitir un determinado sentimiento de belleza.

b) Ecológico: factores o elementos que determinan la calidad del medio natural.

c) Productivo: capacidad de un paisaje para proporcionar beneficios económicos, convirtiendo sus elementos en recursos.

d) Histórico: huellas más relevantes que el ser humano ha dejado en el paisaje a lo largo de la historia.

e) Uso social: utilización, por parte de la sociedad, de un paisaje con finalidades de placer, ocio, paseo, reposo, observación de panorámicas, lugares de encuentro, educación ambiental, práctica de deporte o funciones terapéuticas, entre otras.

f) Mitológico: elementos del paisaje que tienen atribuciones simbólicas colectivas relacionadas con historias fantásticas o leyendas.

g) Religioso y espiritual: corresponden a elementos del paisaje que se relacionan con prácticas y creencias religiosas, como por ejemplo áreas de dólmenes, sepulcros u otros monumentos funerarios.

h) Simbólicos e identitarios: identificación que un determinado colectivo siente con un paisaje. Elementos del paisaje con una gran carga simbólica o identitaria por razones de pertenencia o expresiones de nacionalidad.

A modo de ejemplo de la implementación, en primera instancia, de los indicadores intangibles de paisaje, incluidos en los Catálogos del Paisaje y, finalmente, concretados en valores de paisaje, se reproduce aquí la concreción cartográfica de los valores simbólicos de paisaje correspondientes al Catálogo de Paisaje de las comarcas gerundenses ${ }^{12}$.

Figura $\mathrm{N}^{\circ} 2$

Mapa de valores simbólicos del Catálogo de Paisaje de las Comarcas de Girona
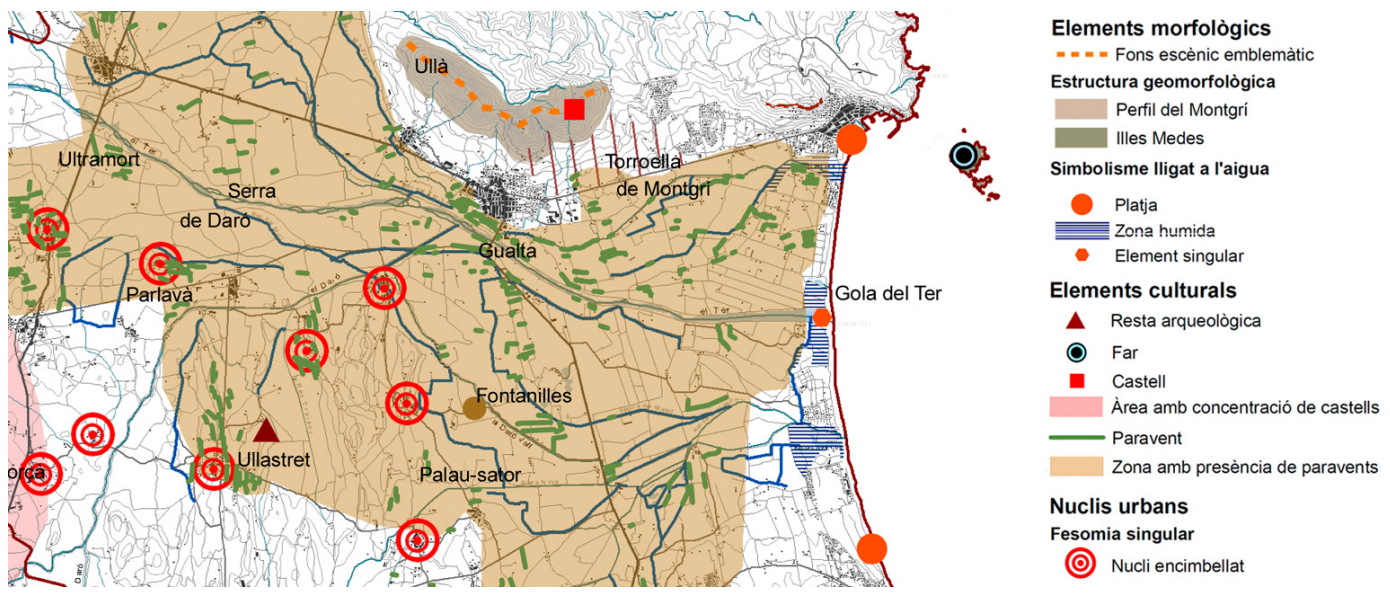

Fuente: Observatorio del Paisaje de Cataluña.

\footnotetext{
12 La implementación de valores intangibles del paisaje de Cataluña se concreta en diversa cartografía (valores sociales, valores estéticos y valores históricos). La documentación completa (informes y cartografía) de todos los Catálogos del Paisaje de Cataluña está disponible en Internet: http://www.catpaisatge.net/esp/catalegs_presentats_G.php
} 


\section{Procesos de identificación de valores tangibles e intangibles del paisaje}

En este apartado se pretende ahondar en el debate relacionado con las características más destacadas de los procesos de identificación de valores tangibles e intangibles del paisaje por parte de los equipos de trabajo del Observatorio del Paisaje de Cataluña. De entrada, debe reseñarse que, tal y como afirman Nogué et al. (2016), las diferencias de percepción existentes respecto al paisaje dificultan la definición de un método cuantitativo de valoración de la calidad de un paisaje. Hay que tener presente que no todos los paisajes tienen el mismo significado para todos $y$, por otra parte, que a cada paisaje se le pueden atribuir diferentes valores y en grados diferentes, según el agente o individuo que lo percibe. Hay que aceptar, por tanto, la existencia de dificultades metodológicas y de diferencias perceptivas respecto al paisaje que dificultan, de entrada, la definición de un método cuantitativo de valoración de la calidad de un paisaje que sea válido y aceptado por todos. Por el mismo motivo expresado antes, de nuevo Nogué, Sala y Grau (2016), sostienen que los catálogos evitan la jerarquización de niveles de calidad del paisaje, tarea muy complicada, por no decir imposible, ya que la mayoría de los valores responden a percepciones o sensaciones de la población subjetivas e inconmensurables.

En lo referido a las fuentes de información a partir de las cuales identificar los valores tangibles e intangibles del paisaje, se ha recurrido a fuentes documentales, fuentes orales, conocedores del territorio (contactos informales con agentes locales y gente que reside en el territorio) y a la herencia artística y literaria del lugar (pintura, fotografía, grabados, postales, etc.)

Capítulo aparte merecen los procesos de participación pública encaminados a determinar los valores tangibles e intangibles de un paisaje. La mayoría de los valores responden a percepciones o sensaciones de la población y, por tanto, son subjetivos y muy difíciles de cuantificar. Para identificar estos valores más perceptivos e interpretativos (como los estéticos, los de uso social o los simbólicos) y, por tanto, de valoración más subjetiva, es muy importante conocer la opinión de la población que vive en el territorio. Por este motivo, los catálogos de paisaje han compatibilizado el rigor científico con la participación de la población y de los principales agentes. Esta participación ha sido básica para identificar muchos de los valores del paisaje que son imperceptibles si se analizan exclusivamente los datos científicos (Nogué et al., 2016).

La valoración estética del paisaje a menudo va asociada a los colores, los olores, los sonidos o los fenómenos meteorológicos. Los valores simbólicos (entre los que se incluyen los identitarios), que en ocasiones emergen con dificultad, aparecieron a menudo en los procesos de participación cuando se buscaba un nombre para la unidad de paisaje, o para lugares concretos. La toponimia y el nombre que la población adjudica a algunos paisajes contiene una elevada carga de valores simbólicos e identitarios. La participación también sirvió para identificar elementos del paisaje que tienen atribuciones mitológicas ligadas a historias fantásticas o a leyendas. Los valores perceptivos están relacionados con la percepción sensorial y emocional que se tiene de un paisaje determinado. En los procesos participativos, y a modo de ejemplo, se definió la Alta Garrotxa como una tierra áspera, salvaje, inhóspita, mientras que la Plana del Empordà se describió como un paisaje tranquilo, equilibrado, acogedor, familiar, amable y pacífico (Nogué et al., 2010). 


\section{Conclusiones}

Cataluña cuenta con una gran riqueza y variedad de paisajes, de lo más elevados de Europa, impregnados de valores de todo tipo. Sin embargo, la mayoría de indicadores actualmente disponibles centran su atención en la evaluación de la biodiversidad o el desarrollo sostenible, basados en la conservación de los ecosistemas y la reducción de los impactos que sobre éstos generan las actividades humanas. En su conjunto, y con contadas excepciones, tienen un carácter marcadamente parcial, ambiental, no suficientemente paisajístico, por el hecho de que no abordan directamente la manera de percibir el paisaje que tiene una sociedad.

En contraposición, la propuesta de indicadores impulsada por el Observatorio del Paisaje de Cataluña propone un diálogo disciplinario sin complejos: que lo cartesiano, medible o ilusoriamente exacto interaccione con lo simbólico, etéreo o pretendidamente difuso. Ello se traduce en la habilitación de indicadores que sean capaces de evaluar el uso social del paisaje, que supone tener en cuenta el grado de satisfacción que produce un determinado paisaje, el nivel de conocimiento que tiene la sociedad o el valor económico que se le atribuye, aspectos, en su conjunto, que la población considera importantes y que dependen de percepciones colectivas.

Aunque hay algunos precedentes en Europa en lo referido a la evaluación inmaterial de los paisajes ${ }^{13}$, parece que el énfasis se sigue depositando en las estructuras de paisaje en detrimento de otros aspectos del paisaje vinculados a su percepción y/o representación por parte de la ciudadanía. En este sentido, el estudio de caso del Observatorio del Paisaje de Cataluña deviene especialmente relevante y significativo, en base a un manifiesto impulso y fomento de la evaluación intangible del paisaje, en consonancia con la nueva cultura del paisaje y del territorio que emerge en Europa a escala internacional, en la cual se otorga cada vez más importancia a las dimensiones perceptivas y sociales asociadas a indicadores de paisaje.

El estudio de caso muestra, además, una aproximación cuantitativa y cualitativa en la evaluación del paisaje, que pretende ser, al mismo tiempo, transversal en el proceso de catalogación de los paisajes de Cataluña en toda su dimensión y características. Sin duda, la evaluación de la dimensión perceptiva del paisaje va estrechamente ligada a la subjetividad, lo que constituye, per se, un obstáculo para una ágil disposición de indicadores, especialmente por la inconmensurabilidad de la mayoría de las percepciones o sensaciones de la población.

El debate en torno al establecimiento de indicadores de paisaje con capacidad para evaluar sus características, valores y/o atributos de forma transversal continúa plenamente vigente. Son muchos los autores que defienden la imperiosa necesidad de establecer indicadores que permitan ir más allá de una métrica de paisaje entendida como una subdisciplina de la ecología del paisaje ocupada, únicamente, en evaluar al paisaje en sus vertientes morfológica y medioambiental, obviando así su naturaleza de constructo social y, por tanto, su carácter marcadamente cultural, histórico, visual y socioeconómico (Bruni, 2016).

El programa de seguimiento de la percepción y apreciación del paisaje en los Países Bajos y los indicadores ingleses de tranquilidad de un paisaje (Tanquillity Maps) y de calidad del paisaje rural (Country Quality Counts). 
No se trata, sin embargo, de un debate fácil. Los indicadores aportan la información necesaria para llevar a término una determinada acción política, por lo que se trata de una información muy sensible. Precisamente por ello, si no se recogen bien, pueden conducir a una determinada política al desastre más absoluto. Los indicadores de carácter intangible y vinculados a las representaciones sociales son, en este sentido, especialmente delicados. Si la información recogida no es de extraordinaria calidad y no se obtiene con un mínimo de consenso científico su utilidad será más que cuestionada.

Los indicadores de paisaje se encuentran en la interfície entre la ciencia y la gestión, entre la generación de conocimiento y la práctica política. Por tanto, se puede considerar que los indicadores son válidos si sirven para tomar buenas decisiones. Asimismo, la implantación de un conjunto de indicadores no debe limitarse a la presentación de una relación de variables, sino que deben incorporarse con normalidad al sistema de toma de decisiones con relación, en este caso, a las políticas de paisaje que tienen incidencia sobre el paisaje de Cataluña.

\section{Referencias bibliográficas}

BOOTH, R. y VALDÉS, C. De la naturaleza al paisaje. Los viajes de Francisco Vidal Gormaz en la coIonización visual del sur de Chile en el siglo XIX. En: Anales del IAA, Vol. 46, No 2, 2016, p.199-216.

BRU, J. Què són i què pretenen els indicadors? In: NOGUÉ J.; PUIGBERT, L y BRETCHA, G. (editores). Indicadors de paisatge. Reptes i perspectives. Olot: Observatori del Paisatge de Catalunya, 2009, p. 18-33.

BRUNI, D. Landscape quality and sustainability indicators. Agriculture and Agricultural Science Procedia, 2016, Vol. 8, p. 698-705.

BUREL, F. \& BAUDRY, J. Landscape ecology: concepts, methods, and applications. Enfield: Science Publishers, 2003.

COBB, C.W. \& RIXFORD, C. Lessons learned from the history of social indicators. San Francisco: Redefining Progress, 1998.

CHECA-ARTASU, M.M. y SUNYER MARTÍN, P. (editores.). El paisaje: reflexiones y métodos de análisis. Universidad Autónoma Metropolitana, 2017.

DALE, V.H. \& KLINE, K.L. Issues in using landscape indicators to assess land changes. Ecological Indicators, 2013, Vol. 28, p. 91-99.

DAVIDSON, J.; SMITH, M.M. \& BONDI, L. (editores.). Emotional geographies. Aldershot: Ashgate Publishing, Ltd., 2012.

ESTÉBANEZ, J. Tendencias y problemática actual de la geografía. Madrid: Cincel, 1989.

FARINA, A. Principles and methods in landscape ecology: towards a science of the landscape. Dordrecht: Springer, 2006. 
FOLCH, R. Diccionario de socioecología. Barcelona: Planeta, 1999.

FRAZIER, A.E. \& KEDRON, P. Landscape Metrics: Past Progress and Future Directions. Current Landscape Ecology Reports, 2017, Vol. 2, No 3, p. 63-72.

GALLOPIN, G. Los indicadores de desarrollo sostenible: aspectos conceptuales y metodológicos. En: Seminario de expertos sobre indicadores de sostenibilidad en la formulación y seguimiento de políticas (4-6 de octubre). Santiago de Chile: FODEPAL, 2006.

LANG, S.; WALZ, U.; KLUG, H.; BLASCHKE, T. \& SYRBE R.U. Landscape metrics-A toolbox for assessing past, present and future landscape structures. In: BENDER, O; EVELPIDOU, N.; KREK, A \& VASSILOPOULOS A. (editores). Geoinformation Technologies for Geocultural Landscapes: European Perspectives. London: Taylor \& Francis, 2008, p. 207-234.

LORENZ, G.; FIGUEROA, M.E; GIANNUZZO, A.N. y LUDUEÑA, M.E. (editores). El paisaje entre ciencia, educación y planificación: el legado que dejamos. Santiago del Estero: Universidad Nacional de Santiago del Estero y Asociación Argentina de Ecología de Paisajes, 2017.

LLAUSÀS, A. \& NOGUÉ, J. Indicators of landscape fragmentation: The case for combining ecological indices and the perceptive approach. Ecological Indicators, 2012, Vol. 15, p. 85-91.

NADAL, J. Presentacions. In: NOGUÉ J; PUIGBERT, L. \& BRETCHA G. (editores). Indicadors de paisatge. Reptes i perspectives. Olot: Observatori del Paisatge de Catalunya, 2009, p. 6-7.

NOGUÉ, J. y SALA, P. Prototipo de Catálogo de Paisaje. Bases conceptuales, Metodológicas y procedimentales para la elaboración de los Catálogos del Paisaje de Cataluña. Olot: Observatorio del Paisaje de Cataluña, 2006.

NOGUÉ, J. Presentacions. In: NOGUÉ J; PUIGBERT, L. \& BRETCHA G. (editores). Indicadors de paisatge. Reptes i perspectives. Olot: Observatori del Paisatge de Catalunya, 2009, p. 14-17.

NOGUÉ, J.; PUIGBERT, L.; SALA, P. y BRETCHA, G. (editores). Paisatge i participación ciutadana. Barcelona: Generalitat de Catalunya, 2010.

NOGUÉ, J. y SAN EUGENIO, J. La dimensión comunicativa del paisaje. Una propuesta teórica y aplicada. Revista de Geografía Norte Grande, 2011, No 49, p. 25-43.

NOGUÉ, J.; SALA, P. \& GRAU, J. Els catàlegs de paisatge de Catalunya. Metodologia. Olot: Observatori del Paisatge de Catalunya y ATLL, 2016.

NÚÑEZ, A; SÁNCHEZ, R. y ARENAS, F. (editores). Fronteras en movimiento e imaginarios geográficos. La cordillera de los Andes como espacialidad sociocultural. Santiago de Chile: Instituto de Geografía-Pontificia Universidad Católica de Chile y RiL editores, 2013.

NÚÑEZ, A; ZUSMAN, P. y HEVILLA, M.C. La producción social de las fronteras (Des-naturalizando las fronteras políticas en América Latina). Revista de Geografía Norte Grande, No 66, 2017, p. 5-8. 
O'NEILL, R.V; RIITTERS, K.H. \& WICKHAM, J.D. Landscape Pattern Metrics and Regional Assessment. Ecosystem Health, 1999, Vol. 5, No 4, p. 225-233.

PARK, C. \& ALLABY, M.A. Dictionary of Environment and Conservation. Oxford: Oxford University Press, 2017.

PASTOR, G.; RODRIGUES ALVES, M.; SANCHEZ FUENTES, D.; MARCHIONNI, F.; y TORRES, L. Miradas e instrumentos para la catalogación de paisajes latinoamericanos. Perspectivas emergentes. Revista de Urbanismo, № 34, 2016, p.138-157.

PENHOS, M. Las fotografías del Álbum de Encina, Moreno y Cía (1883) y la construcción de la Patagonia como espacio geográfico y paisaje. Huellas, 2016, № 9, p.65-80.

PRIORE, R. Presentacions. In: NOGUÉ J; PUIGBERT, L. y BRETCHA G. (editores). Indicadors de paisatge. Reptes i perspectives. Olot: Observatori del Paisatge de Catalunya, 2009, p. 10-12.

RIITTERS, K.H; O'NEILL, RV; HUNSACKER, C.T.; WICKHAM, J.D.; YANKEE, D.H.; TIMMINS, S.P.; JONES, K.B. \& JACKSON, B.L. A factor analysis of landscape pattern and structure metrics". Landscape Ecology, 1995, Vol. 10, No 1, p. 23-39.

SALA, P. Els indicadors de paisatge de Catalunya. In: NOGUÉ J.; PUIGBERT, L.; Gemma BRETCHA (editores). Indicadors de paisatge. Reptes i perspectives. Olot: Observatori del Paisatge de Catalunya, 2009, p. 110-131.

SILVESTRI, G. El lugar común. Una historia de las figuras del paisaje en el Río de la Plata. Buenos Aires: Edhasa, 2011.

TUAN, Y.F. Humanistic geography. Annals of the Association of American Geographers, 1976, Vol. $66, N^{\circ} 2$, p. 266-276.

TURNER, M.G.; GARDNER, R.H. \& O'NEILL, R.V. Landscape Ecology in Theory and Practice. Pattern and Process. New York: Springer, 2001.

UUEMAA, E.; MANDER, Ü. \& MARJA R. Trends in the use of landscape spatial metrics as landscape indicators: a review. Ecological Indicators, 2013, Vol. 28, p. 100-106.

VALDÉS, C. La medida de lo sublime. La Cordillera de los Andes vista desde Chile durante el siglo XIX. Concinnitas, 2012, Vol. 2, No 21, p.139-168.

VALENCIA, M. Tensiones entre procesos de patrimonialización y modernización neoliberal. El caso de los paisajes culturales modernos: conjuntos habitacionales y barrios obreros en América Latina en el siglo XX. Revista de Urbanismo, 2017, No 37, p. 3-16.

VILA, J.; VARGA, D.; LLAUSÀS, A. y RIBAS, A. Conceptos y métodos fundamentales en ecología del paisaje (landscape ecology). Una interpretación desde la geografía. Documents d'Anàlisi Geogràfica, 2006, Vol. 48, p. 151-166. 
YACUZZI, E. El estudio de caso como metodología de investigación: teoría, mecanismos causales, validación, Inomics, 2005, Vol. 1, p. 296-306.

ZARAGOZÍ, B.; BELDA, A; LINARES, J; MARTÍNEZ-PÉREZ, J.E.; NAVARRO, J.T. y ESPARZA, J. A free and open source-programming library for landscape metrics calculations. Environmental Modelling \& Software, 2012, Vol. 31, p. 131-140.

ZUSMAN, P.B. Tierras para el Rey. Tres fronteras y la construcción colonial del territorio del Río de la Plata (1750-1790). Barcelona: Universitat Autònoma de Barcelona, 2000. 
